\title{
Global analysis of differential luminal epithelial gene expression at mouse implantation sites
}

\author{
Ying Chen, Hua Ni, Xing-Hong Ma, Shi-Jun Hu, Li-Ming Luan, Gang Ren, Yue-Chao Zhao, \\ Shi-Jie Li, Hong-Lu Diao, Xiu Xu, Zhen-Ao Zhao and Zeng-Ming Yang
}

College of Life Sciences, Northeast Agricultural University, Harbin 150030, China

(Requests for offprints should be addressed to Z-M Yang; Email: zmyang@neau.edu.cn)

\begin{abstract}
Although implantation types differ between species, the interaction between blastocyst trophectoderm and apical surface of the uterine epithelium is a common event during the implantation process. In this study, uterine luminal epithelium at implantation and inter-implantation sites was isolated by enzymatic digestion and used for microarray analysis. In a mouse microarray containing 12345 unigenes, we found 136 genes upregulated more than twofold at the implantation site, while 223 genes were downregulated by at least twofold. Reverse transcription-PCR was used to verify the differential expression of seven upregulated and six downregulated genes chosen randomly from our microarray analysis, and the expression trends were similar. The differential expression patterns of eight upregulated genes were verified by in situ hybridization. Compared with the inter-implantation site on day 5 of pregnancy and the uterus on day 5 of pseudopregnancy, protease, serine, 12 neurotrypsin, endothelin-1, $\gamma$-glutamyl hydrolase, Ras homolog gene family, member U, T-cell immunoglobulin, and mucin domain containing 2, proline-serine-threonine phosphatase-interacting protein 2, 3-monooxgenase/tryptophan 5-monooxgenase activation protein, $\gamma$-polypeptide, and cysteine-rich protein 61 (Cyr61) were upregulated in the luminal epithelium at implantation site on day 5 of pregnancy. These genes may be related to embryo apposition and adhesion during embryo implantation. Cyr61, a gene upregulated at the implantation site, was chosen to examine its expression and regulation during the periimplantation period by in situ hybridization. Cyr61 mRNA was specifically localized in the luminal epithelium surrounding the implanting blastocyst at day 4 midnight and on day 5 of pregnancy, and in the activated uterus, but not expressed in inter-implantation sites and under delayed implantation, suggesting a role for Cyr61 in mediating embryonic-uterine dialog during embryo attachment. Our data could be a valuable source for future study on embryo implantation.
\end{abstract}

Journal of Molecular Endocrinology (2006) 37, 147-161

\section{Introduction}

Although reproduction is critical to species survival, the process of achieving and maintaining pregnancy is relatively inefficient. Maximal fecundity, the probability of conception during one menstrual cycle, is approximately 30\% (Zinaman et al. 1996). Impaired uterine receptivity is one of the major reasons for the failure of assisted reproductive techniques (Edwards 1995, Spandorfer \& Rosenwaks 1999). Defects in implantation and trophoblast invasion are presently considered the major challenges for the establishment of pregnancy (Herrler et al. 2003).

Depending on the species, trophoblast penetration of the endometrium may remain superficial or continue deep into the endometrium. Nonetheless, all the implantation processes between species involve the interaction between the blastocyst trophectoderm and the apical surface of the uterine luminal epithelium (Carson et al. 2000). Normally, the apical surface of the luminal epithelium is non-adhesive. However, the uterine transition from pre-receptive to receptive requires fundamental structural and functional changes in epithelial cell organization (Kimber \& Spanswick 2000), allowing for successful blastocyst attachment.

Implantation is a complex process involving spatiotemporally regulated endocrine, paracrine, autocrine, and juxtacrine modulators that span cell-cell and cellmatrix interactions. The successful implantation of an embryo depends upon cellular and molecular dialog between the uterus and the embryo (Dey et al. 2004). To date, although many specific factors have been identified during the implantation period, the molecular mechanism of embryo implantation is still unknown. Oligonucleotide microarray analysis has shed insight into a wide range of developmental, oncological, and pharmacological processes by allowing the simultaneous and quantitative assessment of expression levels of thousands of genes (Bethin et al. 2003). Recently, microarray analysis has been used to investigate temporal and spatial gene expression profiles in the mouse uterus during the implantation period (Yoshioka et al. 2000, Reese et al. 2001). 
However, whole uterine fragments were used in these studies. In the uterus, the luminal epithelium represents about $5-10 \%$, the stroma $30-35 \%$, and the myometrium $60-65 \%$ of the major uterine cell types (Finn \& Porter 1975). It is difficult to characterize the specific gene expression in the luminal epithelium because of the interference from large amounts of uterine stroma and myometrium. Yoon et al. (2004) used laser capture microdissection (LCM) to isolate the luminal epithelium at implantation and interimplantation sites for microarray analysis. Although luminal epithelium could be collected accurately by LCM, the mRNA count of luminal epithelium collected from frozen sections is very limited and needs to be amplified several times prior to microarray analysis. Because of the sensitivity in mRNA amplification, artificial errors could be a problem during data analysis. In this study, we used enzymatic digestion to isolate luminal epithelium from the mouse uterus at implantation and inter-implantation sites, of over 100 mice. The luminal epithelium obtained by this method was directly used for mRNA extraction and microarray analysis without any amplification. Differential gene expression profile in uterine luminal epithelium at implantation site was performed using a mouse microarray containing 12345 unigenes. Microarray data were confirmed by both reverse transcription (RT)-PCR and in situ hybridization.

\section{Materials and methods}

\section{Animals and treatments}

Mature mice (Kunming White outbred strain) were caged in a controlled environment with a ratio of $14 \mathrm{~h}$ light: $10 \mathrm{~h}$ darkness cycle. All animal procedures were approved by the Institutional Animal Care and Use Committee of Northeast Agricultural University. Adult females were mated with fertile or vasectomized males of the same strain to induce pregnancy or pseudopregnancy respectively (day $1=$ day of vaginal plug). Pregnancy on days 1-4 was confirmed by recovering embryos from the reproductive tracts. The implantation sites on the day 4 midnight $(2400 \mathrm{~h})$ and on day 5 were identified by i.v. injection of $0.1 \mathrm{ml} 1 \%$ Chicago blue (Sigma) in $0.85 \%$ sodium chloride.

To induce delayed implantation, pregnant mice were ovariectomized under ether anesthesia between 0830 and $0900 \mathrm{~h}$ on day 4 of pregnancy. Progesterone ( $1 \mathrm{mg} /$ mouse) was injected to maintain delayed implantation, from days 5 to 7 . Estradiol-17 $\beta$ (25 ng/mouse) was given to progesterone-primed delayed-implantation mice to terminate delayed implantation. The mice were sacrificed to collect uteri $24 \mathrm{~h}$ after estrogen treatment. Implantation sites were identified by i.v. injection of Chicago blue solution. Delayed implantation was confirmed by flushing the blastocysts from one horn of the uterus.

Hormonal treatments were initiated 2 weeks after mature female mice were ovariectomized. Controls received $0 \cdot 1 \mathrm{ml}$ of sesame oil per mouse. For hormonal treatments, the ovariectomized mice were treated with an injection of estradiol-17 $\beta$ (100 ng/mouse; Sigma), progesterone ( $1 \mathrm{mg} / \mathrm{mouse}$; Sigma), or a combination of the same doses of progesterone and estradiol- $17 \beta$ for $24 \mathrm{~h}$. All steroids were dissolved in sesame oil and injected subcutaneously.

\section{Isolation of uterine luminal epithelium}

Pregnant mice on day $5(0800-0830 \mathrm{~h})$ of pregnancy were used to collect implantation and inter-implantation sites by tail vein injection of Chicago sky blue. For microarray analysis, the uterine fragments with the strongest blue staining on day 5 of pregnancy were collected for implantation sites, and the blue dye-free areas between implantation sites for inter-implantation sites.

Luminal epithelium of mouse uterus was isolated as previously described (Bigsby et al. 1986, Tan et al. 2004) and slightly modified. Uteri at implantation and interimplantation sites were split longitudinally and incubated in $5 \mathrm{ml} 1 \%(\mathrm{w} / \mathrm{v})$ trypsin and $6 \mathrm{mg} / \mathrm{ml}$ dispase in HBSS solution (Gibco) at $4{ }^{\circ} \mathrm{C}$ for $1 \mathrm{~h}, 20^{\circ} \mathrm{C}$ for $1 \mathrm{~h}$, and $37^{\circ} \mathrm{C}$ for $10 \mathrm{~min}$ respectively. After brief shaking, luminal epithelial cells were collected from the supernatants with a mouth-controlled pipette under a stereomicroscope. After enzymatic digestion, implanted blastocysts were found in the digestion solution. To avoid the contamination of glandular epithelium, detached blastocysts, and suspended cells, only the pieces in a single layer were collected. In order to remove any attached uterine stromal cells, the supernatants were centrifuged for $5 \mathrm{~min}$ at $200 \mathrm{~g}$. Then the cell pellets were resuspended in HBSS and spun twice. Finally, the pellets containing uterine luminal epithelial cells were pooled for RNA extraction with TRIZOL reagent (Sigma).

To examine the purity of isolated uterine luminal epithelial cells, an aliquot of cell pellets prior to RNA extraction was resuspended in DMEM/F12 medium $(1: 1$; Sigma) with $10 \%(\mathrm{v} / \mathrm{v})$ fetal bovine serum (Gibco) and plated on a $35 \mathrm{~mm}$ culture dish. After the cells reached around $80 \%$ confluence, they were washed three times in PBS and fixed in cold methanol for $10 \mathrm{~min}$. The cells were blocked with $1 \%(\mathrm{w} / \mathrm{v})$ BSA in PBS for $1 \mathrm{~h}$ and incubated in goat anti-cytokeratin antibody (Santa Cruz Biotechnology, Inc., Santa Cruz, CA, USA) at $4{ }^{\circ} \mathrm{C}$ overnight. After washing three times in PBS, the cells were incubated with biotinylated rabbit anti-goat IgG at $37^{\circ} \mathrm{C}$ for $30 \mathrm{~min}$ and straptavidin conjugated with alkaline phosphatase for $30 \mathrm{~min}$, followed by three washes in PBS. The cells were then 
incubated in Vector Red substrate (Vector Laboratories, Burlingame, CA, USA). Positive reaction products were indicated by red color. Based on cytokeratin immunostaining, the purity of the uterine luminal epithelial cells was approximately $99 \%$.

\section{Microarray analysis}

Uterine luminal epithelium was isolated from implantation and inter-implantation sites as described above respectively. More than 100 mice were used for this study. After uterine luminal epithelium were isolated and extracted for RNA with TRIZOL reagent, reverse transcription, probe labeling, and hybridization were performed according to the procedures from Biostar Biotech, Inc. (Shanghai, China). Briefly, RNA samples extracted with TRIZOL reagent were labeled with Cy3 or Cy5 by direct incorporation of Cy3-dUTP or Cy5dUTP in the reverse transcription reaction, i.e. Cy3 for inter-implantation sites and Cy5 for implantation sites. Labeled samples from implantation and inter-implantation sites were co-hybridized with Genechip BiostarM141s containing 12345 unigenes with defined functions (Biostar Biotech, Inc.). After hybridization at $42{ }^{\circ} \mathrm{C}$ for $16 \mathrm{~h}$, the genechip was washed and air-dried. The fluorescent intensities of Cy3 and Cy5 were scanned with ScanArray 4000 (Packard Bioscience, Billerica, MA, USA) and analyzed with GenePix Pro 3.0 software (Axon Instruments, Foster City, CA, USA). The genes having Cy5:Cy3 ratio within 0 1-10 were chosen for further analysis. In order to eliminate the systematic variation, the Cy3 value of each gene was normalized using a normalization factor $(0 \cdot 976)$, which was the mean value of $\log _{2}(\mathrm{Cy} 5$ intensity/Cy3 intensity). The ratio of each gene expression was calculated as Cy5 intensity over normalized Cy3 intensity. The criterion of twofold difference was used to choose the up- and downregulated genes for the following analysis as described previously (Yang et al. 2002).

\section{RT-PCR}

RNA was extracted from isolated luminal epithelium of both implantation and inter-implantation sites, using TRIZOL reagent. RNA samples were then treated with RQ1 DNase I (Promega) to remove any genomic DNA contamination, extracted with phenol/chloroform, precipitated, and dissolved in formamide at a final concentration of $1 \mu \mathrm{g} / \mu \mathrm{l}$ and stored at $-70^{\circ} \mathrm{C}$.

Total RNAs were reverse-transcribed in a reaction volume of $20 \mu \mathrm{l}$ containing 22 units of RT and $2.5 \mu \mathrm{M}$ oligo-dT using a BcaBEST RNA PCR kit (Takara Biotechniques, Dalian, China). The intensity of amplified bands was scanned with UVP Laboratory Imaging and Analysis System (UVP, Inc., Upland, CA, USA).
Primers, annealing temperatures, and expected product sizes for these genes are listed in Table 1 . Band densities for each gene were normalized to glyceraldehyde-3-phosphate dehydrogenase (Gapdh) expression and the ratios determined. The amplification cycle number of each gene was chosen based on our previous tests to ensure that the amplification was terminated during the exponent phase. RT-PCR for each gene was repeated on, at least, three different samples. The RT-PCR product of each gene was verified by sequencing. Statistical analysis was performed with SPSS software (SPSS Inc., Chicago, IL, USA).

\section{In situ hybridization}

Total RNAs from pregnant mouse uteri were reversetranscribed and amplified with the corresponding primers for each gene listed in Table 1. The amplified fragment of each gene was recovered from agarose gel and cloned into pGEM-T plasmid (pGEM-T Vector System 11; Promega). The cloned fragments were further verified by sequencing. The recombinant plasmid was amplified individually with the primers for T7 and SP6 to prepare templates for labeling sense or antisense probe respectively. Digoxigenin (DIG)labeled antisense or sense cRNA probe was transcribed in vitro using a DIG RNA labeling kit (Roche).

Uteri were cut into 4-6 $\mathrm{mm}$ pieces and flash frozen in liquid nitrogen. Frozen sections $(10 \mu \mathrm{m})$ were mounted on 3-aminopropyltriethoxy silane (Sigma)-coated slides and fixed in $4 \%$ paraformaldehyde solution in PBS. The sections were washed twice in PBS, treated in $1 \%$ Triton-100 for $20 \mathrm{~min}$, and again washed three times in PBS. Following pre-hybridization in the $50 \%$ formamide $/ 5 \times$ SSC solution $(1 \times$ SSC is $0.15 \mathrm{M}$ sodium chloride, $0 \cdot 015 \mathrm{M}$ sodium citrate) at room temperature for $15 \mathrm{~min}$, the sections were hybridized as previously described ( $\mathrm{Ni}$ et al. 2002). Endogenous alkaline phosphatase activity was inhibited with $2 \mathrm{mM}$ levamisole (Sigma). All the sections were counter-stained with $1 \%$ methyl green. The positive signal was visualized as a dark brown color. In situ hybridization for each gene was repeated at least three times with the uteri from three different animals at each stage.

\section{Results}

\section{Microarray analysis}

In order to examine the differential gene expression profile in uterine luminal epithelium on day 5 of pregnancy in the mouse, luminal epithelium was isolated from uterine stroma, glandular epithelium, myometrium, and serosa by enzymatic digestion on day 5 of pregnancy at implantation or inter-implantation 
Table 1 Primer sequences and anticipated product lengths used for reverse transcriptase (RT)-PCR and in situ hybridization



sites respectively. Figure 1 showed the structure of mouse uterus on day 5 of pregnancy at implantation site stained with hematoxylin and eosin, showing that the blastocyst was already attached on the luminal epithelium, which was still intact at the implantation site. During enzymatic digestion, the implanting blastocysts were detached from luminal epithelium, since the blastocysts were already found in the digestion solution. Additionally, a single layer of luminal epithelium was collected with a mouth-controlled pipette under a stereomicroscope to avoid the contamination of blastocysts, stromal cells, and glandular epithelium during the enzymatic digestion.

The differential gene expression profile in the luminal epithelium isolated from mouse uteri between implantation and inter-implantation sites was analyzed by a microarray containing 12345 unigenes. Totally
136 genes were upregulated and 223 genes downregulated by at least twofold in implantation site compared with the inter-implantation site. The upand downregulated genes with known functions are listed in Tables 2 and 3 respectively. Among the upregulated genes, 24 were upregulated by at least threefold, with protease, serine, 12 neurotrypsin (Prss12) being the most dramatically upregulated gene $(6 \cdot 75$-fold). Prss 12 , also called motopsin, was highly expressed in the mouse brain (Iijima et al. 1999). Only 27 genes were downregulated by at least threefold. The highest change among downregulated genes with known function was $\mathrm{N}$-acetylneuraminate pyruvate lyase $(N p l)$ by $5 \cdot 26$-fold.

Among the significantly upregulated genes, most genes had functions related to catalytic activity $(19 \cdot 2 \%)$, protein binding $(12 \cdot 0 \%)$, nucleic acid binding $(8 \cdot 8 \%)$, 


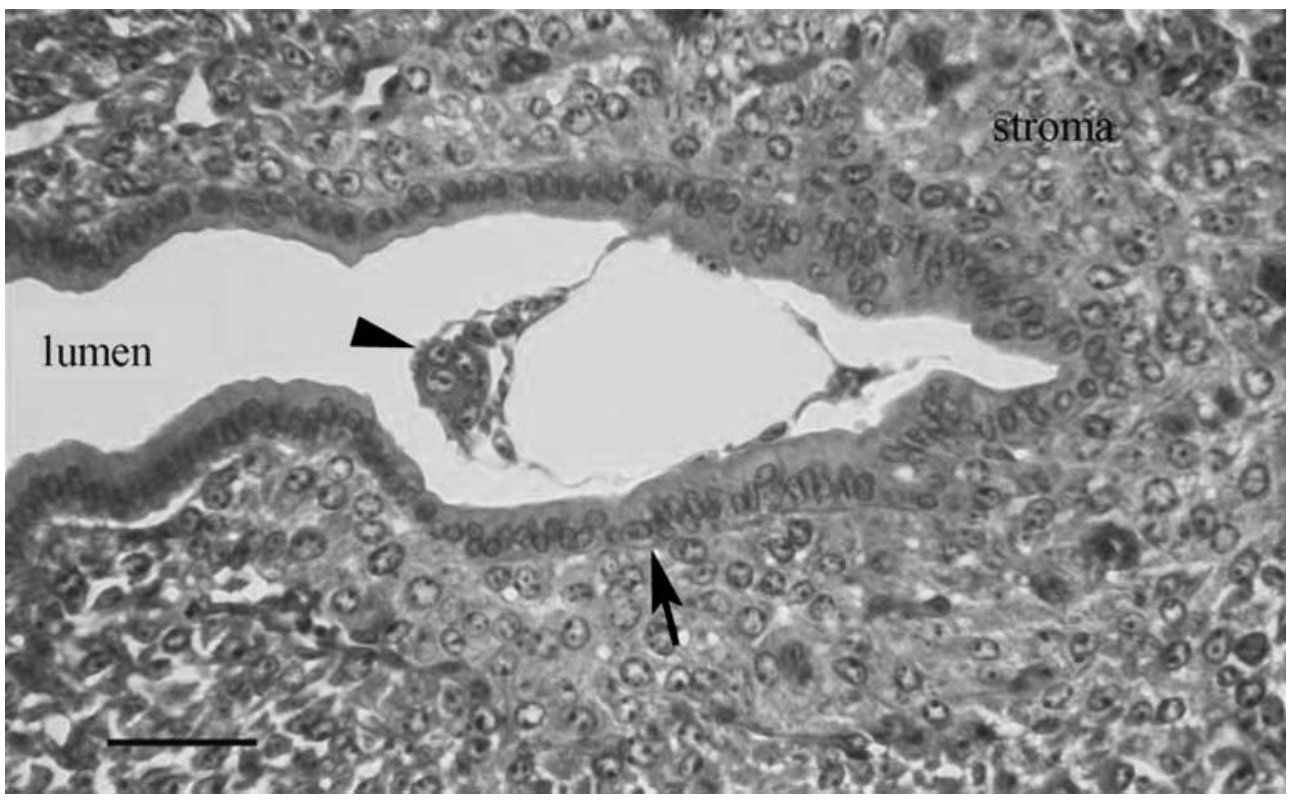

Figure 1 A hematoxylin-eosin staining of mouse uterus at implantation site on day 5 of pregnancy, showing that the blastocyst attached on the luminal epithelium. Arrow: luminal epithelium; arrowhead: blastocyst; bar $=25 \mu \mathrm{m}$.

transporter activity $(7 \cdot 2 \%)$, and hydrolase activity $(6 \cdot 4 \%)$. Based on the molecular functions, the percentages and categories of the downregulated genes were similar to that of upregulated genes. According to the categories of biological processes, both up- and downregulated genes are mainly related to metabolism, cell communication, cell growth and/or maintenance, signal transduction, and transport. There are no significant differences among the up- and downregulated genes. Nevertheless, $5 \cdot 6 \%$ (seven genes) of the upregulated genes are related to cell adhesion, whereas only $1.7 \%$ (three genes) of the downregulated genes were related to cell adhesion. Furthermore, $3 \cdot 2 \%$ (four genes) of the upregulated genes were involved in blood vessel development, while none of the downregulated genes were related to this function. After these genes were classified according to cellular components, $24.0 \%$ (30 genes) of the upregulated genes were extracellular components, whereas $8.7 \%$ (15 genes) of the downregulated genes were related to this function.

\section{Confirmation by RT-PCR}

To verify the data from microarray analysis, we randomly chose seven upregulated and six downregulated genes to examine their expression patterns by semi-quantitative RT-PCR. After the expression of each gene was normalized to Gapdh expression, the ratio between implantation and inter-implantation sites showed similar expression trends from both RT-PCR and microarray analysis, but the expression ratio between them did not completely match between RT-PCR and microarray analysis (Fig. 2). The ratio from microarray analysis was slightly higher than that from RT-PCR except for $\mathrm{Npl}$, which was decreased by 5·26-fold, in microarray, but $6 \cdot 66$ in RT-PCR.

\section{Confirmation by in situ hybridization}

In order to further verify the microarray data, seven upregulated genes were randomly chosen to examine their expression on day 5 of pregnancy and pseudopregnancy by in situ hybridization. Compared with the inter-implantation site on the day of pregnancy and the uterus on day 5 of pseudopregnancy, all of these seven genes were upregulated in the luminal epithelium at implantation site on day 5 of pregnancy (Fig. 3). However, their expression patterns were slightly different. Prss 12 was strongly expressed in the glandular epithelium and the luminal epithelium near the blastocyst (Fig. 3A). Endothelin-1 $(E d n 1)$ is a 21-amino acid vasoconstrictor peptide originally isolated from endothelial cells (Rubanyi \& Polakoff 1994). Edn1 was highly detected in the luminal epithelium surrounding the implanting blastocyst (Fig. 3D). $\gamma$-Glutamyl hydrolase $(G g h)$, a lysosomal endopeptidase, was identified as a biomarker for pulmonary neuroendocrine tumors (He et al. 2004). $G g h$ was strongly expressed in all the luminal epithelium 
Table 2 Genes upregulated by at least twofold at implantation sites

\begin{tabular}{|c|c|c|c|}
\hline & Accession & Fold & Function \\
\hline \multicolumn{4}{|l|}{ Description } \\
\hline Prss12 & NM_008939 & $6 \cdot 75$ & Chymotrypsin activity \\
\hline Pla2g7 & NM_013737 & $4 \cdot 80$ & $\begin{array}{l}\text { 1-Alkyl-2-acetylglycerophosphocholine } \\
\text { esterase activity }\end{array}$ \\
\hline Insulin-like growth factor-binding protein 4 (Igfbp4) & BC019836 & $4 \cdot 36$ & $\begin{array}{l}\text { Insulin-like growth factor-binding; receptor } \\
\text { activity }\end{array}$ \\
\hline Mouse Cyr61 & NM_010516 & $3 \cdot 78$ & Cell adhesion molecule \\
\hline Fibrinogen A $\alpha$ polypeptide & BC005467 & $3 \cdot 56$ & Unspecified \\
\hline Secretory leukocyte protease inhibitor (S/pi) & NM_011414 & $3 \cdot 53$ & Peptidase activity \\
\hline Kininogen $(K n g)$ & NM_023125 & $3 \cdot 48$ & Cysteine protease inhibitor \\
\hline Idb3 & NM_008321 & $3 \cdot 33$ & Transcription corepressor \\
\hline $\begin{array}{l}\text { Cerebellum postnatal development associated protein } 2 \\
\text { (Cadps2) }\end{array}$ & AF000969 & $3 \cdot 32$ & Protein binding \\
\hline Kruppel-like factor 4 (gut; KIf4) & NM_010637 & $2 \cdot 90$ & Transcription factor activity \\
\hline N-myc downstream regulated 1 (Ndr1) & NM_010884 & $2 \cdot 84$ & Unspecified \\
\hline Glutaredoxin 1 (thioltransferase; Glrx1) & NM_053108 & $2 \cdot 78$ & Electron transporter activity \\
\hline S100 calcium-binding protein A6 (calcyclin; S100a6) & NM_011313 & $2 \cdot 59$ & Calcium ion binding \\
\hline Partial mRNA for putative F-box protein 4 (Fbx4) & AJ300659 & $2 \cdot 58$ & Unspecified \\
\hline Neural cell adhesion molecule 1 & X15052 & $2 \cdot 56$ & Cell adhesion molecule \\
\hline Extracellular matrix protein $1(\mathrm{Ecm} 1)$ & NM_007899 & $2 \cdot 49$ & Carrier activity \\
\hline Gap junction membrane channel protein $\beta 1$ (Gjb1) & NM_008124 & $2 \cdot 47$ & Connexon channel activity \\
\hline $\begin{array}{l}\text { Potassium intermediate/small conductance calcium-activated } \\
\text { channel, subfamily } \mathrm{N} \text {, member } 2 \text { (Kcnn2) }\end{array}$ & NM_080465 & $2 \cdot 45$ & Calmodulin binding \\
\hline$\gamma$-Glutamyl hydrolase $(G g h)$ & NM_010281 & $2 \cdot 44$ & $\gamma$-Glutamyl hydrolase \\
\hline Pla2g7 & NM_013737 & $2 \cdot 39$ & $\begin{array}{l}\text { 1-Alkyl-2-acetylglycerophosphocholine } \\
\text { esterase activity }\end{array}$ \\
\hline Aldehyde dehydrogenase (ALDH3B1) & AF362571 & $2 \cdot 29$ & Oxidoreductase activity \\
\hline Tangerin (LOC 114601) & NM_053252 & $2 \cdot 27$ & Actin binding \\
\hline Timd2 & BC028829 & $2 \cdot 26$ & Unspecified \\
\hline Gap junction membrane channel protein $\alpha 5$ (Gja5) & NM_008121 & $2 \cdot 25$ & Connexon channel activity \\
\hline Sorting nexin 2 (Snx2) & NM_026386 & $2 \cdot 21$ & Protein transporter activity \\
\hline Hypothetical protein BC005730 (LOC230822) & NM_145555 & $2 \cdot 21$ & Unspecified \\
\hline Proprotein convertase subtilisin/kexin type 5 & D17583 & $2 \cdot 19$ & Subtilase activity \\
\hline Histidine ammonia lyase ( $\mathrm{Hal})$ & NM_010401 & $2 \cdot 18$ & Ammonia ligase activity \\
\hline Fatty acid desaturase 3 (Fads3) & NM_021890 & $2 \cdot 15$ & Oxidoreductase activity \\
\hline Catenin $\alpha 2$ (Catna2) & NM_009819 & $2 \cdot 15$ & Cell adhesion molecule \\
\hline Glucose-6-phosphatase, transport protein 1 (G6pt1) & NM_008063 & $2 \cdot 14$ & Sugar porter activity \\
\hline Purine-nucleoside phosphorylase (Pnp) & NM_013632 & $2 \cdot 13$ & Phosphorylase activity \\
\hline Pyruvate kinase liver and red blood cell $(P k / r)$ & NM_013631 & $2 \cdot 13$ & Magnesium ion binding \\
\hline Rhou & NM_133955 & $2 \cdot 12$ & GTP binding \\
\hline Neuroblastoma, suppression of tumorigenicity $1(\mathrm{Nb} / 1)$ & NM_008675 & $2 \cdot 12$ & Unspecified \\
\hline B-cell translocation gene 3 (Btg3) & NM_009770 & $2 \cdot 11$ & Transcription factor activity \\
\hline Pstpip2 & NM_013831 & $2 \cdot 11$ & Actin binding \\
\hline Sulfide quinone reductase-like (yeast; Sqrdl) & NM_021507 & $2 \cdot 10$ & Disulfide oxidoreductase activity \\
\hline Angiogenin (Ang) & NM_007447 & $2 \cdot 09$ & Nucleic acid binding \\
\hline Serine (or cysteine) proteinase inhibitor, clade $\mathrm{H}$ (heat shock & NM_009825 & $2 \cdot 09$ & Heat shock protein activity \\
\hline
\end{tabular}
protein 47), member 1 (Serpinh1) 
Table 2 Continued

\section{Description}

Mgst1

Actin-related protein 8 homolog (S. cerevisiae; Actr8)

Tubby like protein 4 (Tulp4)

Degenerative spermatocyte homolog (Drosophila; Degs)

Cfh

Guanosine monophosphate reductase (Gmpr)

$N$-Deacetylase/ $N$-sulfotransferase (heparin glucosaminyl) 4

(Ndst4)

$\mathrm{V}$-abl Abelson murine leukemia oncogene 1

Malic enzyme, supernatant (Mod1)

Syndecan 1 (Sdc1)

Protein kinase $\mathrm{C}$ and casein kinase substrate in neurons 2

(Pacsin2)

Nucleosome-binding protein 1 (Nsbp1)

\begin{tabular}{|c|c|c|}
\hline Accession & Fold & Function \\
\hline NM_019946 & $2 \cdot 07$ & Glutathione transferase activity \\
\hline NM_027493 & $2 \cdot 07$ & Structural constituent of cytoskeleton \\
\hline NM_054040 & $2 \cdot 06$ & Unspecified \\
\hline NM_007853 & $2 \cdot 05$ & Metallopeptidase activity \\
\hline NM_009888 & $2 \cdot 05$ & Complement activity \\
\hline NM_025508 & $2 \cdot 02$ & GMP reductase activity \\
\hline NM_022565 & $2 \cdot 01$ & Sulfotransferase activity \\
\hline J02995 & $2 \cdot 01$ & ATP binding \\
\hline NM_008615 & $2 \cdot 01$ & $\begin{array}{l}\text { Malate dehydrogenase (decarboxylating) } \\
\text { activity }\end{array}$ \\
\hline NM_011519 & $2 \cdot 00$ & Cytoskeletal protein binding \\
\hline NM_011862 & $2 \cdot 00$ & Cytoskeletal protein binding \\
\hline NM_016710 & $2 \cdot 00$ & Chromatin binding \\
\hline
\end{tabular}

except for that surrounding the implanting blastocyst (Fig. 3G). A low level of $G g h$ expression was also seen in the luminal epithelium at inter-implantation site (Fig. 3H), but not in day 5 pseudopregnant uterus (Fig. 3I). Ras homolog gene family member U (Rhou; Santin et al. 2005) was detected in both luminal epithelium and subluminal stromal cells on day 5 of pregnancy at implantation site (Fig. 3J), but not at interimplantation site on day 5 of pregnancy (Fig. 3K), and on day 5 of pseudopregnancy (Fig. 3L). T-cell immunoglobulin and mucin domain containing 2 (Timd2) is critical for the regulation of Th2 responses during autoimmune inflammation (Chakravarti et al. 2005). Timd2 was detected mainly in the luminal epithelium surrounding the implanting blastocyst (Fig. 3M). Proline-serine-threonine phosphatase-interacting protein 2 (Pstpip2) regulates F-actin bundling, and enhances filopodia formation and motility in macrophages (Chitu et al. 2005). A low level of Pstpip2 expression was observed in all the luminal epithelium at implantation site (Fig. 3P). 3-Monooxgenase/tryptophan 5-monooxgenase-activation protein, $\gamma$-polypeptide ( $Y w h a g$ ), also called 14-3-3 $\gamma$, is a member of 14-3-3 family (Takahashi 2003). Ywhag expression was strongly detected in the luminal epithelium surrounding the implanting blastocyst and weakly in the subluminal stromal cells (Fig. 3S).

\section{Expression and regulation of cysteine-rich protein 61 (Cyr61) in mouse uterus}

In our microarray analysis, Cyr61 was upregulated by $3 \cdot 77$ at the implantation site. Cyr61 is also known as CCN1 (CCN is the initial of Cyr61, connective tissue growth factor ( $C t g f)$, and nephroblastoma-overexpressed,
NOV) and belongs to the CGN family (Perbal 2001). Because Cyr61 was highly expressed in human endometrium and upregulated in the endometria of women with endometriosis (Absenger et al. 2004, Punyadeera et al. 2005), Cyr61 was then chosen to verify the expression pattern and to examine its expression during early pregnancy, pseudopregnancy, delayed implantation, and steroid hormonal treatments by in situ hybridization. There was no detectable Cyr61 expression in the uterus from days 1 to 3 of pregnancy (data not shown). Cyr61 expression was not detected in the mouse uterus on the morning of day 4 of pregnancy (Fig. 4A), whereas it was observed in the luminal epithelium immediately surrounding the blastocyst on day 4 midnight when the attachment reaction was just initiated (Fig. 4B). On day 5 of pregnancy, when embryo implantation occurred, Cyr61 mRNA signals were seen only in the luminal epithelium of mouse uterus at the implantation site (Fig. 4C), but not at the inter-implantation site (Fig. 4D). Furthermore, Cyr61 signals were not detected in the mouse uterus on day 5 of pseudopregnancy (Fig. 4E) or during delayed implantation (Fig. 4G). After delayed implantation was terminated by estrogen treatment and the embryos implanted, Cyr61 mRNA expression was once again observed in the luminal epithelium (Fig. 4H), expression similar to that observed at the implantation sites on day 5 of pregnancy. After Cyr61 anti-sense probe was replaced with Cyr61 sense probe, there was no detectable signal in the uterus on day 5 of pregnancy (Fig. 4F). Additionally, there was no detectable Cyr61 mRNA signal in the uterus of ovariectomized mice. After ovariectomized mice were treated with either estrogen or progesterone for $24 \mathrm{~h}$, Cyr61 mRNA expression was not seen in the uterus (data not shown). 
Table 3 Genes downregulated by at least twofold at implantation sites

Accession

NM 028749

Description

$\mathrm{Npl}$

Calbindin-28K (Calb1)

Bach

Glutathione S-transferase, $\mu 1$ (Gstm1)

Slc6a6

Myd88

Interleukin 1 receptor, type I (//1r1)

Neuronal differentiation related protein $(N d r p)$

Peripheral myelin protein, $22 \mathrm{kDa}$ (Pmp22)

ATPase, $\mathrm{Ca}^{++}$transporting, plasma membrane 2 (Atp2b2)

Sulfotransferase family $1 \mathrm{~A}$, phenol-preferring, member 1 (Sult1a1)

Gstm2

Arylsulfatase A

Aldehyde dehydrogenase family 7 , member A1 (Aldh7a1)

Progressive ankylosis (Ank)

Son cell proliferation protein (Son)

Tmsb4x

ATP-binding cassette, subfamily G (WHITE), member 1 (Abcg1)

Glutathione S-transferase, $\mu 6$ (Gstm6)

Uracil DNA glycosylase (Ung)

Zmpste24

Complement component 4-binding protein

(C4bp)

Syntaxin 18 (Stx18)

Low density lipoprotein receptor-related protein associated protein 1

Odd homeobox 1 protein (Ob1)

Interferon regulatory factor 1 (Irf1)

CGI-74-like SR-rich (LOC192196)

Lymphocyte antigen 75 ( Ly75)

Rho-associated coiled-coil forming kinase 2 (Rock2)

Nucleolar protein 5 (Nol5)

Tropomodulin 2 (Tmod2)

6-Pyruvoyl-tetrahydropterin synthase (Pts)

$\alpha$ Glucosidase 2, $\alpha$ neutral subunit (G2an)

Creatine kinase, muscle $(C \mathrm{kmm})$

Duffy blood group (Dfy)

Endothelial differentiation, lysophosphatidic

acid G-protein-coupled receptor 7 (Edg7)

Phospholipid scramblase 1 (PIscr1)

Growth factor receptor bound protein 2 (Grb2)

Hypothetical protein (MGC27617)

Profilin 2 (Pfn2)

Dihydrolipoamide branched chain transacylase E2 $(D b t)$

RAB4B, member RAS oncogene family (Rab4b)

Survivor of motor neuron protein interacting

protein 1 (Sip1)

Creatine kinase, mitochondrial 1 , ubiquitous

(Ckmt1)

Actinin $\alpha 3$ (Actn3)

Glutathione S-transferase, $\alpha 4$ (Gsta4)

Polymerase (DNA directed), $\delta 2$, regulatory

subunit (50 kDa; Pold2)

Kruppel-type zinc finger protein KROX-25

Solute carrier family $15(\mathrm{H}+$ /peptide transporter), member 2 (Slc15a2)

Mitochondrial ribosomal protein L45 (Mrp/45)
$-5 \cdot 26$

$-4 \cdot 57$

$-4 \cdot 35$

$\begin{array}{ll}\text { NM_010358 } & -4 \cdot 10 \\ \text { NM_009320 } & -3 \cdot 85\end{array}$

$\begin{array}{ll}\text { NM_010358 } & -4 \cdot 10 \\ \text { NM_009320 } & -3 \cdot 85\end{array}$

BC005591 $\quad-3.61$

NM $008362 \quad-3.38$

NM_031879 $-3 \cdot 14$

NM $008885-3.08$

NM_009723 $\quad-2.99$

NM_133670

NM_008183

X73230

$-2 \cdot 99$

$-2 \cdot 99$

$-2 \cdot 74$

$-2 \cdot 70$

$-2 \cdot 70$

$-2 \cdot 65$

NM_020332

NM 019973

NM_021278

NM 009593

NM 008184

NM_011677

AY029194

NM_007576

NM_026959

D00622

AF492703

NM_008390

NM_138680

NM 013825

NM_009072

NM_018868

NM_016711

NM_011220

NM 008060

NM_007710

NM 010045

NM_022983

NM_011636

NM 008163

NM_144813

NM 019410

NM_010022

NM_029391

NM 025656

NM 009897

NM 013456

NM_010357

NM 008894

AF281634

NM_021301

NM 025927
$-2 \cdot 64$

$-2 \cdot 61$

$-2 \cdot 54$

$-2 \cdot 47$

$-2 \cdot 47$

$-2 \cdot 46$

$-2 \cdot 46$

$-2 \cdot 43$

$-2 \cdot 39$

$-2 \cdot 35$

$-2 \cdot 33$

$-2 \cdot 29$

$-2 \cdot 25$

$-2 \cdot 23$

$-2 \cdot 22$

$-2 \cdot 18$

$-2 \cdot 17$

$-2 \cdot 16$

$-2 \cdot 15$

$-2 \cdot 15$

$-2 \cdot 12$

$-2 \cdot 11$

$-2 \cdot 09$

$-2.09$

$-2.08$

$-2 \cdot 07$

$-2 \cdot 06$

$-2 \cdot 05$

$-2 \cdot 05$

$-2 \cdot 05$

$-2.04$

$-2 \cdot 03$

$-2 \cdot 02$

$-2 \cdot 02$

$-2 \cdot 00$
Function

$\mathrm{N}$-Acetylneuraminate lyase activity

Calcium ion binding

Palmitoyl-CoA hydrolase activity

Glutathione transferase activity

$\beta$-Alanine transporter activity

Transmembrane receptor activity

Activating receptor activity

Unspecified

Structural constituent of eye lens

ATP binding

Aryl sulfotransferase activity

Glutathione transferase activity

Cerebroside-sulfatase activity

Aldehyde dehydrogenase (NAD) activity

Inorganic diphosphate transporter activity

DNA binding

Actin binding

ATP binding

Glutathione transferase activity

Uracil DNA $N$-glycosylase activity

Metalloendopeptidase activity

Complement activity

Protein transporter activity

Heparin binding

Transcriptional repressor activity

Transcription factor activity

Nucleic acid binding

Receptor activity

ATP binding

Chaperone activity

F-actin capping activity

Magnesium ion binding

Glucosidase activity

Creatine kinase activity

Rhodopsin-like receptor activity

Lysosphingolipid and lysophosphatidic acid receptor activity

Calcium ion binding

$\mathrm{SH} 3 / \mathrm{SH} 2$ adaptor protein activity

Calcium/potassium/sodium antiporter activity

Actin binding

Acyltransferase activity

GTP binding

Pre-mRNA splicing factor activity

Creatine kinase activity

Actin cross-linking activity

Glutathione transferase activity

DNA-directed DNA polymerase activity

DNA binding

High-affinity oligopeptide transporter activity

Structural constituent of ribosome 


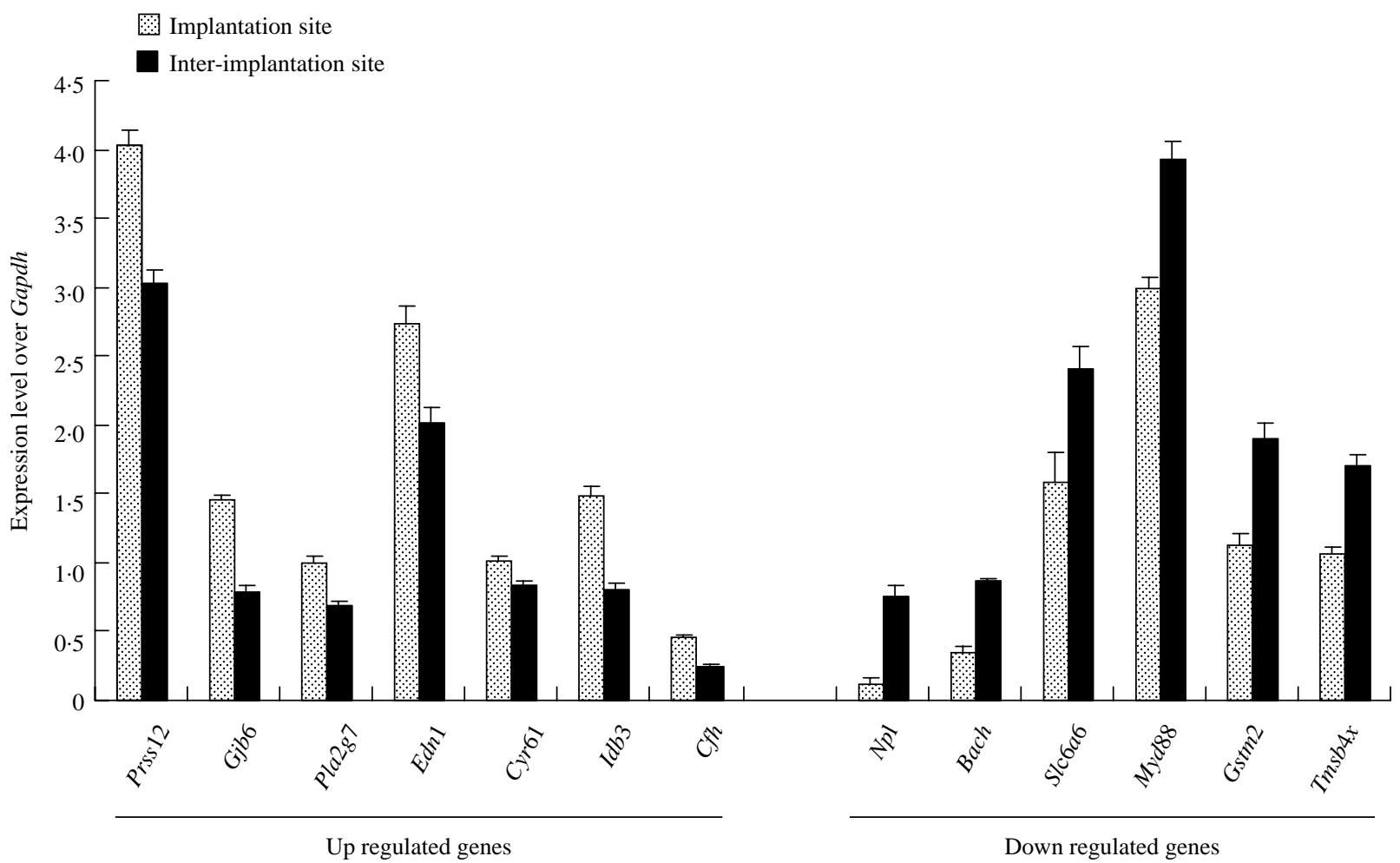

Figure 2 Semi-quantification of reverse transcriptase (RT)-PCR. RNA was extracted using TRIZOL reagent from isolated luminal epithelium on both implantation and inter-implantation sites respectively. To verify the data from microarray analysis, seven upregulated and six downregulated genes were randomly chosen to examine their expression patterns by semi-quantitative RT-PCR. After the expression of each gene was normalized to glyceraldehyde-3-phosphate dehydrogenase expression, the ratio of each gene between implantation site and inter-implantation site was determined. RT-PCR for each gene was repeated with three different samples. Values are presented as the mean \pm S.E.M. $(n=3)$.

\section{Discussion}

Embryo implantation is a process where the activated blastocyst establishes close contact with the uterine luminal epithelium and attaches to initiate implantation. However, the luminal epithelium represents only about $5-10 \%$ of uterine cell types (Finn \& Porter 1975). Although implantation and inter-implantation sites can be distinguished by tail injection of Chicago blue dye solution (Psychoyos 1986), it is very hard to characterize the differential gene expression specifically in the luminal epithelium. Although luminal epithelium at implantation sites could be isolated using LCM, the samples obtained from LCM are very limited and must be amplified several times prior to microarray analysis (Yoon et al. 2004). For the samples collected by LCM, the random error from mRNA amplification and degradation during the staining could pose problems. In our study, the luminal epithelium at both implantation and inter-implantation sites was isolated by enzymatic digestion from over 100 mice and directly used for microarray analysis.
Because the uterus at implantation sites was used in previous microarray studies (Yoshioka et al. 2000, Reese et al. 2001), it was difficult to compare our data with theirs. In the study by Yoon et al. (2004), the luminal epithelium isolated by LCM was used for microarray analysis, and only three identical upregulated genes were detected both by us and by Yoon et al. (2004). Compared with inter-implantation sites, Ggh, inhibitor of DNA-binding 3, and secreted acidic cysteine-rich glycoprotein (Sparc) were increased by $2 \cdot 44-, 3 \cdot 33-$, and $3 \cdot 08$-folds in our study, but by $1 \cdot 63-, 1 \cdot 89-$, and $1 \cdot 48$ folds, by Yoon et al. (2004) respectively. Except for these three genes, other up- or downregulated genes were not shared. The main reason for these differences could be due to the methods for isolating luminal epithelium. We isolated the whole luminal epithelium at implantation or inter-implantation sites by enzymatic digestion, whereas Yoon et al. (2004) collected only the luminal epithelium immediately surrounding the implanting blastocyst. The luminal epithelium immediately surrounding the implanting blastocyst differed greatly from the rest of luminal epithelium in its gene 




Figure 3 Confirmation by in situ hybridization, showing the expression pattern of protease, serine, 12 neurotrypsin, endothelin-1, $\gamma$-glutamyl hydrolase, Ras homolog gene family, member $U$, T-cell immunoglobulin and mucin domain containing 2, proline-serinethreonine phosphatase-interacting protein 2, and 3-monooxgenase/tryptophan 5-monooxgenase-activation protein, $\gamma$-polypeptide in mouse uteri at implantation site and inter-implantation site on day 5 of pregnancy, and on day 5 of pseudopregnancy respectively. Compared to inter-implantation site, the expression level of these seven genes in luminal epithelium was stronger at implantation site. On day 5 of pseudopregnancy, there were no detectable signals for these seven genes. *Implanting blastocyst; bar $=60 \mu \mathrm{m}$. 



Figure 4 Cysteine rich protein 61 expression at implantation site. (A) Day $4(0800 \mathrm{~h})$; (B) day $4(2400 \mathrm{~h})$; (C) day 5 at implantation site; (D) day 5 at inter-implantation site; (E) day 5 of pseudopregnancy; $(F)$ day 5 at implantation site (sense probe for control); (G) delayed implantation; (H) activation of delayed implantation. ${ }^{*}$ Embryo; bar $=60 \mu \mathrm{m}$. 
expression. For example, cyclooxygenase-2 and basigin were specifically expressed in the luminal epithelium surrounding the implanting blastocyst (Lim et al. 1997, Xiao et al. 2002).

\section{Upregulated genes}

In this study, 136 genes were upregulated by at least twofold, of which 24 were upregulated by at least threefold. Prss 12 was the highest one upregulated by $6 \cdot 75$-fold and was strongly expressed in the glandular epithelium and the luminal epithelium near the blastocyst at implantation site on day 5 of pregnancy, but not detected in the inter-implantation site on day 5 of pregnancy, and in the day 5 pseudopregnant uterus. It seems that Prss 12 in the luminal epithelium was specifically induced by implanting blastocyst. Prss 12 , also called motopsin, was highly expressed in mouse brain (Iijima et al. 1999). However, the expression, regulation, and function of this gene in early pregnancy still remain unknown.

Edn1 and Ywhag were strongly expressed in the luminal epithelium surrounding the implanting blastocyst, suggesting a role during embryo apposition and attachment. Edn1 stimulates neutrophil adhesion to endothelial cells by an effect on the expression of adhesive molecules on the neutrophil surface. $E d n 1$ also stimulates neutrophil accumulation in vivo and in vitro in the heart (Lopez Farre et al. 1993). Edn1 induces leukocyte rolling and adherence through a predominantly endothelin receptor A-mediated mechanism in the submucosal venules of the intestinal microcirculation (Boros et al. 1998). Human decidual cells in early pregnancy can synthesize and release EDN1. These cells also possess specific functional receptors for EDN1, which are coupled to phosphoinositide hydrolysis, suggesting a possible role for EDN1 in autocrine and/or paracrine function in human decidual cells (Kubota et al. 1992). Ywhag, also called 143-3 $\gamma$, is a member of the 14-3-3 family. The 14-3-3 family is a large group of highly conserved $30 \mathrm{kDa}$ acidic proteins (Takahashi 2003). 14-3-3 proteins participate in integrin-activated signaling pathways through their interaction with docking protein p130 (Cas), which may contribute to important biological responses regulated by cell adhesion to the extracellular matrix (GarciaGuzman et al. 1999). 14-3-3 $\zeta$ can regulate cell adhesion and spreading through the interaction with ADAM22 (Zhu et al. 2005). Whether Ywhag is involved in cell adhesion still remains unknown.

Timd2 was also highly expressed in the luminal epithelium surrounding the implanting blastocyst. However, there are no papers that mention its role in cell adhesion. The T-cell Ig and mucin domaincontaining gene locus has been linked to differences in $\mathrm{T}(\mathrm{h}) 2$ responsiveness and asthma susceptibility in mice (Encinas et al. 2005). Timd2 is expressed preferentially in differentiated Th2 cells and is critical for the regulation of Th2 responses during autoimmune inflammation (Chakravarti et al. 2005). In normal pregnancy, particularly at the maternal-fetal interface, anti-inflammatory, Th-2, cytokines predominate (Wegmann et al. 1993). An appropriate balance between pro- and anti-inflammatory cytokines is thought to be crucial for determining the success or failure of a pregnancy (Formby 1995).

Rhou belongs to Ras homolog gene family. Ras homolog gene family, member I (ARHI) was downregulated in uterine serous papillary cancer compared with normal endometrial cells (Santin et al. 2005). Ras homolog gene family plays a central role in a wide range of physiological processes, including cell morphology, cell adhesion, cytokinesis, cell motility, and cell growth (Van Aelst \& D'Souza-Schorey 1997, Hall 1998). However, the expression and function of Rhou during early pregnancy still remain to be determined.

Pstpip2, also called macrophage actin-associated tyrosine-phosphorylated protein (MAYP), directly regulates F-actin bundling and enhances filopodia formation and motility in macrophages. Filopodia are postulated to increase directional motility by acting as environmental sensors. The MAYP-stimulated increase in directional movement may be at least partly explained by enhancement of filopodia formation (Chitu et al. 2005). In our study, Pstpip2 was expressed in all luminal epithelium at the implantation site, but not in the inter-implantation site on day 5 of pregnancy, and in the uterus on day 5 of pseudopregnancy, suggesting that Pstpip2 may be involved in embryo apposition and attachment.

\section{Downregulated genes}

In our microarray, 223 genes were downregulated by at least twofold in the luminal epithelium at implantation site, of which nine were decreased by over threefold, including $\mathrm{N}$-acetylneuraminate pyruvate lyase (5·26-fold), calbindin-28K (4-57-fold), brain acyl-CoA hydrolase $(4 \cdot 35$-fold $)$, glutathione S-transferase $\mu 1$ $(4 \cdot 10$-fold $)$, solute carrier family $6(3 \cdot 85$-fold $)$, myeloid differentiation primary response gene $88(3 \cdot 61$-fold $)$, interleukin 1 receptor type 1 (3.38-fold), neuronal differentiation related protein $(3 \cdot 14$-fold $)$, and peripheral myelin protein $22(3 \cdot 08$-fold $)$. Although $\mathrm{N}$ acetylneuraminate pyruvate lyase was greatly reduced at implantation sites, the expression and regulation of this gene is still unknown.

Calbindin-28K was decreased by $4 \cdot 57$-fold at implantation sites in our study. A previous study showed that calbindin-28K protein was increased in the endometrial epithelium just before implantation, but 
disappeared at implantation sites after attachment (Luu et al. 2004). Because calbindin-28K is able to inhibit apoptosis in osteoblastic cells (Bellido et al. 2000 ), the apoptosis caused by the downregulation of calbindin-28K could destabilize the luminal epithelium at the implantation site and facilitate trophoblast invasion (Luu et al. 2004). However, calbindin-28Kdeficient mice do not show implantation failure (Airaksinen et al. 1997). Calbindin-9K mRNA was also specifically downregulated at the implantation site (Nie et al. 2000). Although calbindin-28K-deficient mice are fertile, embryo implantation is blocked if both calbindin-9K and calbindin-28K are inhibited just before implantation (Luu et al. 2004).

\section{A role for Cyr61 in mediating embryo attachment at the implantation site}

Cyr61 is also known as CCN1 (CCN is the initial of Cyr61, Ctgf, and NOV) and belongs to the CCN family. Other members of the CCN family include CCN2 (Ctgf), CCN3 (NOV), and the Wnt-inducible secreted proteins CCN4 (WISP-1), CCN5 (WISP-2), and CCN6 (WISP-3, Perbal 2001). Cyr61 overexpression in the undifferentiated AN3CA endometrial cancer cell line led to decreased cell growth and increased apoptosis in liquid culture. Moreover, the increased apoptosis in these endometrial cancer cells with Cyr61 over expression was associated with elevated expression of the pro-apoptotic proteins $\mathrm{Bax}, \mathrm{Bad}$, and tumor necrosis factor receptor-associated ligand (Chien et al. 2004). In the mouse, uterine epithelial cells surrounding the implanted embryo will undergo apoptotic cell death on day 5 evening or day 6 of pregnancy (Parr et al. 1987, Joswig et al. 2003). It is possible that the Cyr61 expression might be closely related to subsequent apoptosis in the luminal epithelium.

We found that Cyr61 was upregulated by $3 \cdot 77$-fold in the luminal epithelium at implantation sites. Because Cyr61 was specifically induced in the luminal epithelium at the implantation site by the activated blastocyst, it may play a key role during embryo attachment. Cyr61 and Ctgf supported the attachment of endothelial cells through integrin $\alpha \mathrm{v} \beta 3$ (Kireeva et al. 1998) and cell surface heparan sulfate proteoglycans (Chen et al. 2000, 2004). Integrin $\alpha \mathrm{v} \beta 3$ was expressed in the apical pole of the uterine luminal epithelium and on the epithelial surface of blastocyst (Aplin et al. 1996, Lessey et al. 1996). Nevertheless, the normal Mendelian ratio of Cyr61 mutant embryos at E9.5 suggests that Cyr61 is not critical for implantation (Mo et al. 2002). It is possible that Cyr61 function may be compensated by other CCN members. CTGF shared many common functions with Cyr61, including promoting endothelial cell growth, migration, adhesion, and survival (Brigstock et al. 2002). Moreover, CTGF was also expressed in the luminal epithelium at mouse implantation site (Surveyor et al. 1998). In the Ptgs2 null CD-1 mice, Ptgs1 is upregulated and can maintain mouse implantation although cyclooxygenase (COX)-2 is essential for mouse implantation (Wang et al. 2004). However, whether CTGF is upregulated in Cyr61 null mice remains to be determined.

In human endometrium, Cyr61 was more highly expressed during the proliferative than during the secretory phase in endometrium (Punyadeera et al. 2005). Cyr61 was one of the most upregulated genes in endometria of women with endometriosis and in ectopic endometrium (Absenger et al. 2004). Basic fibroblast growth factor, epidermal growth factor, tumor necrosis factor $\alpha$, and interleukin-1 could stimulate Cyr61 expression (Schutze et al. 1998). Furthermore, Cyr61 could upregulate the synthesis of vascular endothelial growth factor (VEGF)-A and VEGF$\mathrm{C}$ to regulate the processes of angiogenesis, inflammation, and matrix remodeling in the context of cutaneous wound healing (Chen et al. 2001, Brigstock 2002). Interestingly, Cyr61 mutation led to impaired Vegf-C expression in the allantoic mesoderm, suggesting that Cyr61-regulated expression of Vegf-C plays a role in vessel bifurcation (Mo et al. 2002). VEGF was strongly expressed in mouse uterus during implantation period and played a key role in regulating vascular permeability and angiogenesis (Chakraborty et al. 1995, Halder et al. 2000). Increased vascular permeability and angiogenesis were crucial to successful implantation, decidualization, and placentation (Dey et al. 2004).

In conclusion, there are 136 genes upregulated and 223 genes downregulated in the luminal epithelium at the implantation site of mouse uterus by at least twofold in this microarray study compared to inter-implantation site. Among these regulated genes, there were 13 genes confirmed by RT-PCR and eight upregulated genes verified by in situ hybridization. Cyr61 is specifically induced in the luminal epithelium at the implantation site by the activated blastocyst and may play a key role during embryo attachment. Our data could be a valuable source for future study on embryo implantation.

\section{Acknowledgements}

We thank Dr Sudhansu K Dey for helpful discussions and critical readings of the manuscript. We also thank Ren-Wei Su, Hao Yu, and Fei Gao for their help in collecting tissues and isolating luminal epithelium. This work was supported by Chinese National Natural Science Foundation grants 30330060, 30500361 and 30570198, and the Special Funds for Major State Basic Research Project (G1999055903). The authors declare that there is no conflict of interest that would prejudice the impartiality of this scientific work. 


\section{References}

Absenger Y, Hess-Stumpp H, Kreft B, Kratzschmar J, Haendler B, Schutze N, Regidor PA \& Winterhager E 2004 Cyr61, a deregulated gene in endometriosis. Molecular Human Reproduction 10 399-407.

Airaksinen MS, Eilers J, Garaschuk O, Thoenen H, Konnerth A \& Meyer M 1997 Ataxia and altered dendritic calcium signaling in mice carrying a targeted null mutation of the calbindin D28k gene. PNAS 94 1488-1493.

Aplin JD, Spanswick C, Behzad F, Kimber SJ \& Vicovac L 1996 Integrins $\beta 5, \beta 3, \alpha \mathrm{v}$ are apically distributed in endometrial epithelium. Molecular Human Reproduction 2 527-534.

Bellido T, Huening M, Raval-Pandya M, Manolagas SC \& Christakos S 2000 Calbindin-D28k is expressed in osteoblastic cells and suppresses their apoptosis by inhibiting caspase-3 activity. Journal of Biological Chemistry 275 26328-26332.

Bethin KE, Nagai Y, Sladek R, Asada M, Sadovsky Y, Hudson TJ \& Muglia LJ 2003 Microarray analysis of uterine gene expression in mouse and human pregnancy. Molecular Endocrinology 17 1454-1469.

Bigsby RM, Cooke PS \& Cunha GR 1986 A simple efficient method for separating murine uterine epithelial and mesenchymal cells. American Journal of Physiology 251 E630-E636.

Boros M, Massberg S, Baranyi L, Okada H \& Messmer K 1998 Endothelin 1 induces leukocyte adhesion in submucosal venules of the rat small intestine. Gastroenterology 114 103-114.

Brigstock DR 2002 Regulation of angiogenesis and endothelial cell function by connective tissue growth factor (CTGF) and cysteinerich 61 (CYR61). Angiogenesis 5 153-165.

Carson DD, Bagchi I, Dey SK, Enders AC, Fazleabas AT, Lessey BA \& Yoshinaga K 2000 Embryo implantation. Developmental Biology 223 217-237.

Chakraborty I, Das SK \& Dey SK 1995 Differential expression of vascular endothelial growth factor and its receptor mRNAs in the mouse uterus around the time of implantation. Journal of Endocrinology 147 339-352.

Chakravarti S, Sabatos CA, Xiao S, Illes Z, Cha EK, Sobel RA, Zheng XX, Strom TB \& Kuchroo VK 2005 Tim-2 regulates T helper type 2 responses and autoimmunity. Journal of Experimental Medicine 202 437-444.

Chen N, Chen CC \& Lau LF 2000 Adhesion of human skin fibroblasts to Cyr61 is mediated through integrin $\alpha 6 \beta 1$ and cell surface heparan sulfate proteoglycans. Journal of Biological Chemistry $\mathbf{2 7 5}$ 24953-24961.

Chen CC, Mo FE \& Lau LF 2001 angiogenic factor Cyr61 activates a genetic program for wound healing in human skin fibroblasts. Journal of Biological Chemistry 276 47329-47337.

Chen N, Leu SJ, Todorovic V, Lam SC \& Lau LF 2004 Identification of a novel integrin alphavbeta3 binding site in CCN1 (CYR61) critical for pro-angiogenic activities in vascular endothelial cells. Journal of Biological Chemistry 279 44166-44176.

Chien W, Kumagai T, Miller CW, Desmond JC, Frank JM, Said JW \& Koeffler HP 2004 Cyr61 suppresses growth of human endometrial cancer cells. Journal of Biological Chemistry 279 53087-53096.

Chitu V, Pixley FJ, Macaluso F, Larson DR, Condeelis J, Yeung YG \& Stanley ER 2005 PCH family member MAYP/PSTPIP2 directly regulates F-actin bundling and enhances filopodia formation and motility in macrophages. Molecular Biology of the Cell 16 2947-2959.

Dey SK, Lim H, Das SK, Reese J, Paria BC, Daikoku T \& Wang H 2004 Molecular cues to implantation. Endocrine Reviews 25 341-373.

Edwards RG 1995 Clinical approaches to increasing uterine receptivity during human implantation. Human Reproduction 10 (suppl 2) $60-66$.

Encinas JA, Janssen EM, Weiner DB, Calarota SA, Nieto D, Moll T, Carlo DJ \& Moss RB 2005 Anti-T-cell Ig and mucin domaincontaining protein 1 antibody decreases TH2 airway inflammation in a mouse model of asthma. Journal of Allergy and Clinical Immunology 116 1343-1349.
Finn CA \& Porter DG 1975 The uterus: cells and tissues of the endometrium. Acton, MA: Publishing Sciences Group, Inc.

Formby B 1995 Immunologic response in pregnancy. Its role in endocrine disorders of pregnancy and influence on the course of maternal autoimmune diseases. Endocrinology and Metabolism Clinics of North America 24 187-205.

Garcia-Guzman M, Dolfi F, Russello M \& Vuori K 1999 Cell adhesion regulates the interaction between the docking protein p130(Cas) and the 14-3-3 proteins. Journal of Biological Chemistry 274 5762-5768.

Halder JB, Zhao X, Soker S, Paria BC, Klagsbrun M, Das SK \& Dey SK 2000 Differential expression of VEGF isoforms and VEGF(164)specific receptor neuropilin-1 in the mouse uterus suggests a role for VEGF (164) in vascular permeability and angiogenesis during implantation. Genesis 26 213-224.

Hall A 1998 Rho GTPases and the actin cytoskeleton. Science $\mathbf{2 7 9}$ 509-514.

He P, Varticovski L, Bowman ED, Fukuoka J, Welsh JA, Miura K, Jen J, Gabrielson E, Brambilla E, Travis WD et al. 2004 Identification of carboxypeptidase $\mathrm{E}$ and gamma-glutamyl hydrolase as biomarkers for pulmonary neuroendocrine tumors by cDNA microarray. Human Pathology 35 1196-1209.

Herrler A, Von Rango U \& Beier HM 2003 Embryo-maternal signalling: how the embryo starts talking to its mother to accomplish implantation. Reproductive Biomedecine Online 6 244-256.

Iijima N, Tanaka M, Mitsui S, Yamamura Y, Yamaguchi N \& Ibata Y 1999 Expression of a serine protease (motopsin PRSS12) mRNA in the mouse brain: in situ hybridization histochemical study. Brain Research. Molecular Brain Research 66 141-149.

Joswig A, Gabriel HD, Kibschull M \& Winterhager E 2003 Apoptosis in uterine epithelium and decidua in response to implantation: evidence for two different pathways. Reproductive Biology and Endocrinology 144.

Kimber SJ \& Spanswick C 2000 Blastocyst implantation: the adhesion cascade. Seminar on Cell and Developmental Biology 11 77-92.

Kireeva ML, Lam SC \& Lau LF 1998 Adhesion of human umbilical vein endothelial cells to the immediate-early gene product Cyr61 is mediated through integrin $\alpha \mathrm{v} \beta 3$. Journal of Biological Chemistry 273 3090-3096.

Kubota T, Kamada S, Hirata Y, Eguchi S, Imai T, Marumo F \& Aso T 1992 Synthesis and release of endothelin-1 by human decidual cells. Journal of Clinical Endocrinology and Metabolism 75 1230-1234.

Lessey BA, Ilesanmi AO, Lessey MA, Riben M, Harris JE \& Chwalisz K 1996 Luminal and glandular endometrial epithelium express integrins differentially throughout the menstrual cycle: implications for implantation, contraception, and infertility. American Journal of Reproductive Immunology 35 195-204.

Lim H, Paria BC, Das SK, Dinchuk JE, Langenbach R, Trzaskos JM \& Dey SK 1997 Multiple female reproductive failures in cyclooxygenase 2-deficient mice. Cell 91 197-208.

Lopez Farre A, Riesco A, Espinosa G, Digiuni E, Cernadas MR, Alvarez V, Monton M, Rivas F, Gallego MJ, Egido J et al. 1993 Effect of endothelin-1 on neutrophil adhesion to endothelial heart. Circulation 88 1166-1171.

Luu KC, Nie GY \& Salamonsen LA 2004 Endometrial calbindins are critical for embryo implantation: evidence from in vivo use of morpholino antisense oligonucleotides. PNAS 101 8028-8033.

Mo FE, Muntean AG, Chen CC, Stolz DB, Watkins SC \& Lau LF 2002 CYR61 (CCN1) is essential for placental development and vascular integrity. Molecular and Cellular Biology 22 8709-8720.

Ni H, Sun T, Ding NZ, Ma XH \& Yang ZM 2002 Differential expression of microsomal PGE synthase at the implantation sites and in the decidual cells in mouse uterus. Biology of Reproduction 67 351-358.

Nie GY, Li Y, Wang J, Minoura H, Findlay JK \& Salamonsen LA 2000 Complex regulation of calcium-binding protein D9k (calbindin$\mathrm{D}(9 \mathrm{k})$ ) in the mouse uterus during early pregnancy and at the site of embryo implantation. Biology of Reproduction 62 27-36. 
Parr EL, Tung HN \& Parr MB 1987 Apoptosis as the mode of uterine epithelial cell death during embryo implantation in mice and rats. Biology of Reproduction 36 211-225.

Perbal B 2001 NOV (nephroblastoma overexpressed) and the CCN family of genes: structural and functional issues. Molecular Pathology 54 57-79.

Psychoyos A 1986 Uterine receptivity for implantation. Annals of the New York Academy of Sciences 476 36-42.

Punyadeera C, Dassen H, Klomp J, Dunselman G, Kamps R, Dijcks F, Ederveen A, de Goeij A \& Groothuis P 2005 Oestrogen-modulated gene expression in the human endometrium. Cellular and Molecular Life Sciences 62 239-250.

Reese J, Das SK, Paria BC, Lim H, Song H, Matsumoto H, Knudtson KL, DuBois RN \& Dey SK 2001 Global gene expression analysis to identify molecular markers of uterine receptivity and embryo implantation. Journal of Biological Chemistry 276 44137-44145.

Rubanyi GM \& Polakoff A 1994 Endothelins: molecular biology, biochemistry, pharmacology, physiology and pathophysiology. Pharmacological Reviews 46 325-415.

Santin AD, Zhan F, Cane' S, Bellone S, Palmieri M, Thomas M, Burnett A, Roman JJ, Cannon MJ, Shaughnessy J Jr et al. 2005 Gene expression fingerprint of uterine serous papillary carcinoma: identification of novel molecular markers for uterine serous cancer diagnosis and therapy. British Journal of Cancer 92 1561-1573.

Schutze N, Lechner A, Groll C, Siggelkow H, Hufner M, Kohrle J \& Jakob F 1998 Human analog of murine cystein rich protein 61 is a 1 $\alpha, 25$-dihydroxyvitamin D3 responsive immediate early gene in human fetal osteoblasts: regulation by cytokines, growth factors, and serum. Endocrinology 139 1761-1770.

Spandorfer S \& Rosenwaks Z 1999 Impact of maternal age and ovarian age on implantation efficacy. In Embryo Implantation: Molecular, Cellular and Clinical Aspects, pp 12-19. Ed DD Carson. New York: Springer-Verlag.

Surveyor GA, Wilson AK \& Brigstock DR 1998 Localization of connective tissue growth factor during the period of embryo implantation in the mouse. Biology of Reproduction 59 1207-1213.

Takahashi Y 2003 14-3-3 proteins: gene, gene expression, and function. Neurochemical Research 28 1265-1273.
Tan Y, Li M, Cox S, Davis MK, Tawfik O, Paria BC \& Das SK 2004 HB-EGF directs stromal cell polyploidy and decidualization via cyclin D3 during implantation. Developmental Biology 265 181-195.

Van Aelst LV \& D'Souza-Schorey C 1997 Rho GTPases and signal networks. Genes and Development 11 2295-2322.

Wang H, Ma WG, Tejada L, Zhang H, Morrow JD, Das SK \& Dey SK 2004 Rescue of female infertility from the loss of cyclooxygenase-2 by compensatory up-regulation of cyclooxygenase- 1 is a function of genetic makeup. Journal of Biological Chemistry 279 10649-10658.

Wegmann TG, Lin H, Guilbert L \& Mosmann TR 1993 Bidirectional cytokine interactions in the maternal-fetal relationship: is successful pregnancy a TH2 phenomenon? Immunology Today 14 353-356.

Xiao LJ, Chang H, Ding NZ, Ni H, Kadomatsu K \& Yang ZM 2002 Basigin expression and hormonal regulation in mouse uterus during the peri-implantation period. Molecular Reproduction and Development 63 47-54.

Yang YH, Dudoit S, Luu P, Lin DM, Peng V, Ngai J \& Speed TP 2002 Normalization for cDNA microarray data: a robust composite method addressing single and multiple slide systematic variation. Nucleic Acids Research 30 e15.

Yoon SJ, Choi DH, Lee WS, Cha KY, Kim SN \& Lee KA 2004 A molecular basis for embryo apposition at the luminal epithelium. Molecular and Cellular Endocrinology 219 95-104.

Yoshioka K, Matsuda F, Takakura K \& Noda Y 2000 Determination of genes involved in the process of implantation: application of GeneChip to scan 6500 genes. Biochemistry and Biophysics Research Communication 272 531-538.

Zhu P, SangY, Xu H, Zhao J, Xu R, Sun Y, Xu T, Wang X, Chen L, Feng H, Li C \& Zhao S 2005 ADAM22 plays an important role in cell adhesion and spreading with the assistance of 14-3-3. Biochemistry and Biophysics Research Communication 331 938-946.

Zinaman MJ, Clegg ED, Brown CC, O’Connor J \& Selevan SG 1996 Estimates of human fertility and pregnancy loss. Fertility and Sterility 65 503-509.

Received in final form 21 May 2006

Accepted 5 June 2006 